\title{
Clinical Dry Eye and Meibomian Gland Features Among Dry Eye Patients in Rural and Urban Ghana
}

This article was published in the following Dove Press journal: Clinical Ophthalmology

\author{
Eugene Appenteng Osae (D) I,2 \\ Reynolds Kwame Ablordeppey' \\ Jens Horstmann ${ }^{2}$ \\ David Ben Kumah' \\ Philipp Steven ${ }^{2,3}$ \\ 'Department of Optometry and Visual \\ Science, Kwame Nkrumah University of \\ Science and Technology, Kumasi, Ghana; \\ ${ }^{2}$ Department of Ophthalmology, Division \\ for Dry-Eye and Ocular GvHD, Medical \\ Faculty, University of Cologne, Cologne, \\ Germany; ${ }^{3}$ Cluster of Excellence: Cellular \\ Stress Responses in Aging-Associated \\ Diseases (CECAD), University of \\ Cologne, Cologne, Germany
}

\begin{abstract}
Aim: To compare differences in clinical dry eye features and meibomian gland health status between dry eye patients from rural and urban populations in Ghana.

Methods: We examined 211 (rural=109, urban=102) participants with subjective dry eye symptoms. Tear film break -up time (TBUT), Schirmer's test and ocular surface staining (OSS) were assessed. Symptoms were evaluated using the SPEED II questionnaire. Meibomian glands (MG) in the right eye upper (UL) and lower lids (LL) were imaged using a custom meibographer. MG area was determined by intensity threshold segmentation using Image J software. MG loss (MGL) was also graded based on Pult's grading scheme. Mann-Whitney, Spearman correlation, chi-square and odds analyses were performed; $\mathrm{p}<0.05$ was considered significant.

Results: Rural participants showed greater SPEED scores, reduced TBUT, and lower Schirmer scores, $\mathrm{p}<0.05$. The proportion of rural participants with MGL were significantly more $(82.3 \%)$ than urban participants $(63.3 \%), p<0.05$. They also showed greater MGL than urban participants, $\mathrm{p}<0.05$. Chi-square test revealed significantly different meiboscale distributions (UL: $\chi^{2}=13.58$, LL: $\chi^{2}=15.29$ ) between the groups, $\mathrm{p}<0.05$. Overall significant relationships were observed between MGL and age $\left[r_{s}=0.61\right]$, OSS $\left[r_{s}=0.35\right]$, TBUT $\left[r_{s}=\right.$ -0.52 ], and Schirmer scores $\left[\mathrm{r}_{\mathrm{s}}=-0.40\right], \mathrm{p}<0.05$.

Conclusion: The data suggest that the participants from the rural population have worse dry eye and meibomian gland health status than those from the urban population. The significant relationships between the various clinical variables suggest important links between MGD and DED. Subtle differences in the everyday working and living environment could likely account for the differences in the severity of DED and MGD between the two groups. And considering the increased pattern of urbanization, industrialization and modernization and the related environmental effects in Africa, future longitudinal studies on specific environmental risk factors or mediators of DED and MGD are necessary to ascertain the MGD and DED situation in Ghana and Africa at large.
\end{abstract}

Keywords: dry eye, meibomian gland dysfunction, ocular surface, dysfunctional tear syndrome

\section{Introduction}

Dry eye disease (DED) synonymous with dysfunctional tear syndrome is a multifactorial disease of the ocular surface accompanied by a variety of clinical signs and discomfort-related symptoms. ${ }^{1}$ These can include redness, pain, itchiness, grittiness, burning sensation and general irritations in the eye. DED can be a source of significant morbidity, affecting normal physical and mental functioning. ${ }^{1,2}$

As a multifactorial condition, DED is driven by multiple etiological players including tear film hyperosmolarity, ocular surface inflammation and damage, neurosensory abnormalities and meibomian gland dysfunction (MGD). ${ }^{1,2}$ MGD
Correspondence: Eugene Appenteng

Osae

Department of Optometry and Visual

Science, Kwame Nkrumah University of

Science and Technology, Private Mail Bag,

Kumasi, Ghana

Tel +233200466 637

Email osaeappen@gmail.com 
affects the quantity and quality of tear film lipids resulting in instability and faster evaporation of the tear film at the ocular surface. ${ }^{3}$ This leads to the evaporative form of DED. Evaporative DED accounts for about $70 \%$ of all DED cases while aqueous-deficient DED or overlapping forms account for the remainder. ${ }^{1-4}$ Other contributors to DED include ageing, desiccating environmental conditions and autoimmune disorders like Sjogren's syndrome and ocular graft-versus-host disease. ${ }^{1,2,5}$

Reported prevalence of DED ranges from $5 \%$ to $50 \%$. This depends on the populations studied and majority of existing reports are from epidemiological data of DED studies conducted in Europe, North America and Asia., Thus, very little information is known about the incidence of DED in Africa. Earlier studies in Africa linked DED to malnutrition (vitamin A deficiency) and trachoma., ${ }^{7} 8$ However, many African countries have over the past two decades seen tremendous improvement in their socio-economic statuses ${ }^{6,9}$. There is some improvement in health care by the elimination of trachoma and vitamin A deficiency through the World Health Organization's SAFE [S= surgery, $\mathrm{A}=$ antibiotics, $\mathrm{F}=$ facial cleanliness, $\mathrm{E}=$ environmental improvement] strategy and nutritional supplementation programs. ${ }^{10}$ In addition to these socioeconomic developments, there are remarkable events of industrialization, urbanization and modernization; many countries in Africa have transitioned into emerging economies. ${ }^{6}$

In our earlier review on the epidemiology of DED in Africa, we highlighted how industrialization, urbanization and modernization could alter the normal environment and contribute to human disease including MGD and DED. ${ }^{6}$ Furthermore, we previously reported a high $(84 \%)$ prevalence of meibomian gland loss (MGL) together with clinical dry eye features in a cross-section of dry eye patients in an urban and industrialized population in Ghana. ${ }^{11}$

As a follow-up, we present herein, our recent findings from a comparative study examining the differences in clinical DED features and meibomian gland health status between dry eye patient groups from an industrialized urban population and non-industrialized rural population in Ghana, where there are obvious differences in the everyday working and living environments.

\section{Methods}

\section{Ethical Considerations}

We adhered to the Declaration of Helsinki. We obtained informed consent from all study participants. Ethical approval was granted by the Committee on Human Research, Publication and Ethics at the School of Medical Sciences, Kwame Nkrumah University of Science and Technology/Komfo Anokye Teaching Hospital, Ghana (CHRPE/AP/448/16).

\section{Study Sites}

This was a two-center study. We chose the Outpatient Eye Clinic/Vision Center of the Optometry Department at the Kwame Nkrumah University of Science and Technology in Kumasi as our urban site and the Westphalian Eye Hospital in Oyoko as our rural site. The two locations are both in the Ashanti region of Ghana which lies in the tropical savannah zone of Africa and have no differences in climate (Figure 1). ${ }^{12,13}$

Kumasi is an urban and heavily industrialized city and the capital of the Ashanti region. It is the second largest and second most populous city in Ghana with an estimated population of 3.3 million people and a density of about 14,000 people/square kilometers. Oyoko is a relatively small agrarian community located $35 \mathrm{~km}$ southeast of Kumasi with a native population of about 5000 and an unstated density that is far below the regional and national estimates. $^{12,13}$

\section{Study Design and Sample}

The study was cross-sectional and clinic based. The minimum sample size estimation was based on our previous study ${ }^{11}$ as follows:

$$
\mathrm{n}=\frac{\left(\mathrm{Z}_{1-\alpha / 2}\right)^{2} \mathrm{P}(1-\mathrm{P})}{\mathrm{d}^{2}}
$$

where $\mathrm{n}$ is the sample size, $Z_{1-\alpha / 2}=1.96$ is the standard normal variate (at $5 \%$ type 1 error $(\mathrm{p}<0.05) ; P=84 \%=0.84$ is expected proportion of persons with MGD and clinical dry eye features based on our previous published study ${ }^{11}$ and $d=5 \%=0.05$ is our decided absolute error or precision. So, from the above, the estimated minimum sample size

$$
=\frac{(1.96)^{2} \times 0.84(1-0.84) \approx 206}{0.05^{2}}
$$

Therefore, we studied a total of 211 (109 urban and 102 rural) participants over a 10 -month period. These participants presented with subjective dry eye complaints at the two eye clinics in the respective locations. Participants were included if they had no history of ocular surgery including eyelid correction and no use of drugs like 


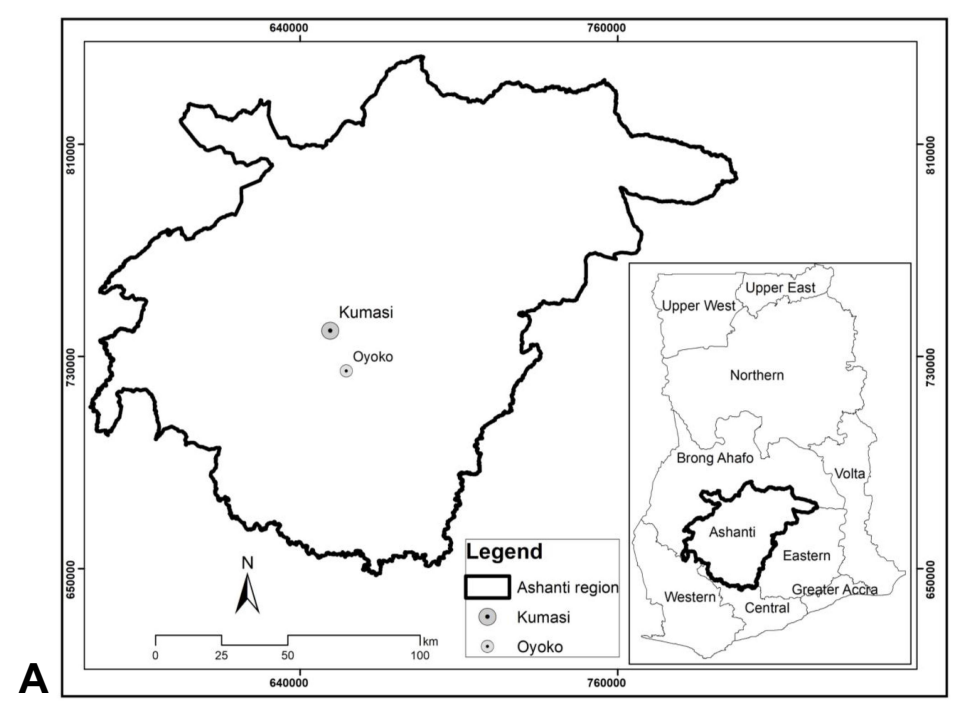

B

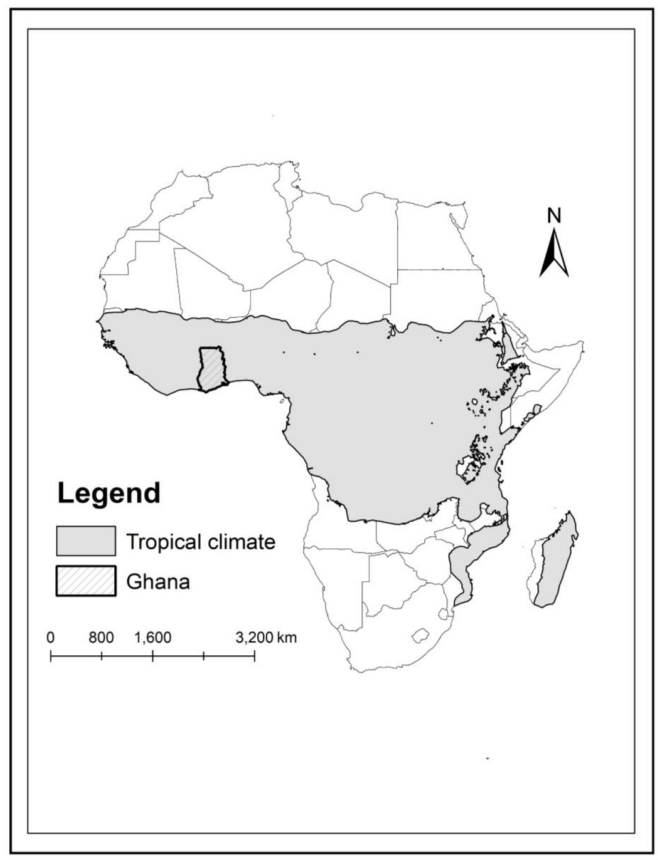

Figure I (A) Map of Ashanti region showing the study sites (Inset: A regional map of Ghana) and (B) map of Africa showing the extent of the tropical climate zone.

isotretinoin nor benzalkonium chloride-containing formulations that could affect the meibomian gland health or induce DED. ${ }^{1,3}$

\section{Clinical Dry Eye Assessment}

We evaluated DED symptoms using the SPEED II questionnaire. This is a four-question and a Likert-scale type survey used to assess the frequency and severity of dry eye symptoms, monitoring diurnal and longer symptom changes over the course of 3 months. ${ }^{14}$ This was followed by a thorough slit lamp examination. Tear film break-up time (TBUT) was measured after application of $2 \%$ topical fluorescein to the ocular surface. Ocular surface staining (OSS) was evaluated under cobalt blue light and Wratten \#12 filter (Kodak, NJ, USA) and graded using the Oxford Grading Scheme as described by Bron et al. ${ }^{15}$ Tear production was assessed by Schirmer I test. ${ }^{11}$
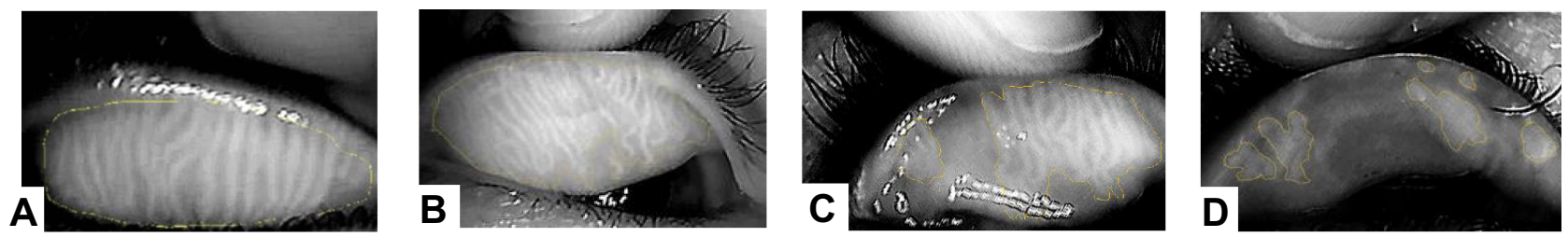

Figure 2 Meibographs of upper eyelids showing (A) Grade 0, (B) Grade I, (C) Grade 2 and (D) Grade 4 of MGL. 


\section{Statistical Analyses}

Data were analyzed using SPSS Statistics version 23. Shapiro-Wilk's test for normality was performed on the data. Differences between groups were evaluated by Mann-Whitney $U$-test; similarities of distributions between groups were inspected graphically and results reported using an exact sampling distribution for $U$. Values are expressed as median [minimum-maximum]. We also performed Spearman correlation, Pearson's chi-square and odds ratio tests to determine the relationship between the various variables and $\mathrm{p}<0.05$ was considered significant.

\section{Results}

\section{Participants' Demographics}

Table 1 shows the distribution of participants by gender and study site. Rural participants were significantly older (48.0 [23.0-72.0] years) than urban participants (43.0 [21.0-75.0] years), $U=4164.5 \mathrm{z}=-3.148, \mathrm{p}<0.01$. Overall female participants were more $(64 \%)$ than male participants $(36 \%)$. Occupational profiles of participants were different between the two study sites. Majority (52\%) of the urban participants worked as civil servants, ie, educationists, medics, bankers and clerks whereas a greater proportion (44\%) of the rural participants were farmers (Table 2).

\section{Clinical Dry Eye Parameters}

Overall, rural participants exhibited more dry eye symptoms with significantly higher total SPEED scores (7.0 [0-15.0]) than urban participants $(5.0[0-15.0]), U=4254.0 \mathrm{z}=-2.955$, $\mathrm{p}<0.01$ (Figure $3 \mathrm{~A}$ ). Tear production as demonstrated by

Table I Distribution of Study Participants by Gender and Study Site

\begin{tabular}{|l|l|l|l|}
\hline \multirow{2}{*}{ Study Site } & \multicolumn{2}{|l|}{ Gender of Participants } & \multirow{2}{*}{ Total } \\
\cline { 2 - 3 } & Male & Female & \\
\hline Urban & 41 & 68 & 109 \\
Rural & 36 & 66 & 102 \\
\hline Total & 77 & 134 & 211 \\
\hline
\end{tabular}

Schirmer readings was significantly lower for rural participants $(15.0[1.0-28.0] \mathrm{mm})$ compared to urban participants $(20.0[3.0-25.0] \mathrm{mm}), \quad U=4969.0 \mathrm{z}=-3.131, \mathrm{p}<0.01$ (Figure 3B).

Furthermore, rural participants showed a significantly greater tear film instability indicated by lower TBUT scores (10.0 [2.0-23.0]s) compared to urban participants (12.0[2.0 24.0]s), $U=4446.5 \mathrm{z}=-2.515, \mathrm{p}<0.05$ (Figure 3C). On one hand, there was no statistically significant difference in fluorescein staining scores between rural and urban participants. The OSS score was low (1.0 [0-2.0]) for both rural and urban participants, $U=4969.0, \mathrm{z}=-1.414, \mathrm{p}>0.05$.

\section{Eyelid Conditions and Meibomian Gland Evaluation}

Slit-lamp examination did not reveal any cases of lid folliculations and scarification worth documenting among the study participants. Only one rural participant had a history of resolved unilateral (left eye) trachoma. The combined cases of (anterior and or/posterior) blepharitis were higher among rural participants (27.4\%) than urban participants (4.6\%). The cases of stye were also higher among rural participants (46.1\%) than urban participants (24.8\%).

Overall, $72.5 \%$ of the 211 participants showed some degree of meibomian gland loss (MGL) of $\geq$ grade1 in upper or lower lids. MGL cases were significantly higher (82.3\%) for rural participants than for urban participants $(63.3 \%), p<0.01$. Further, rural participants showed statistically significant greater MGL than urban participants; upper lid $(25.0 \%$ [0-85.0\%]) vs $(10.0 \%[0-75.5 \%]),[U=4237, \mathrm{z}=$ $-3.00, \mathrm{p}<0.01]$ and lower lid $(25.0 \%$ [0-80.0\%8] vs $(5.0 \%$ [0-60\%], $[U=3533, z=-4.64, p<0.001]$ (Figure 4A and B).

The odds of having MGL and a history of blepharitis was higher for rural participants than urban participants. Rural participants with a history of blepharitis had $78 \%$ higher odds $(\mathrm{OR}=1.78$ [95\% CI, 0.47-6.77]) of having MGL than those without a history of blepharitis, $p>0.05$. On one hand, urban participants with a history of blepharitis had $93 \%$ lower odds $(\mathrm{OR}=0.07$ [95\% CI, 0.01-0.12]) of having

Table 2 Distribution of Study Participants by Occupation and Study Site

\begin{tabular}{|l|l|l|l|l|l|l|l|}
\hline \multirow{2}{*}{ Study Site } & \multicolumn{4}{l}{ Occupation of Participants } & \multicolumn{4}{l|}{ Total } \\
\cline { 2 - 8 } & Artisan & Civil Servant & Farmer & Student & Trader & Unemployed \\
\hline $\begin{array}{l}\text { Urban } \\
\text { Rural }\end{array}$ & 3 & 57 & 1 & 24 & 18 & 6 & 109 \\
\hline Total & 10 & 6 & 45 & 14 & 20 & 7 & 102 \\
\hline
\end{tabular}



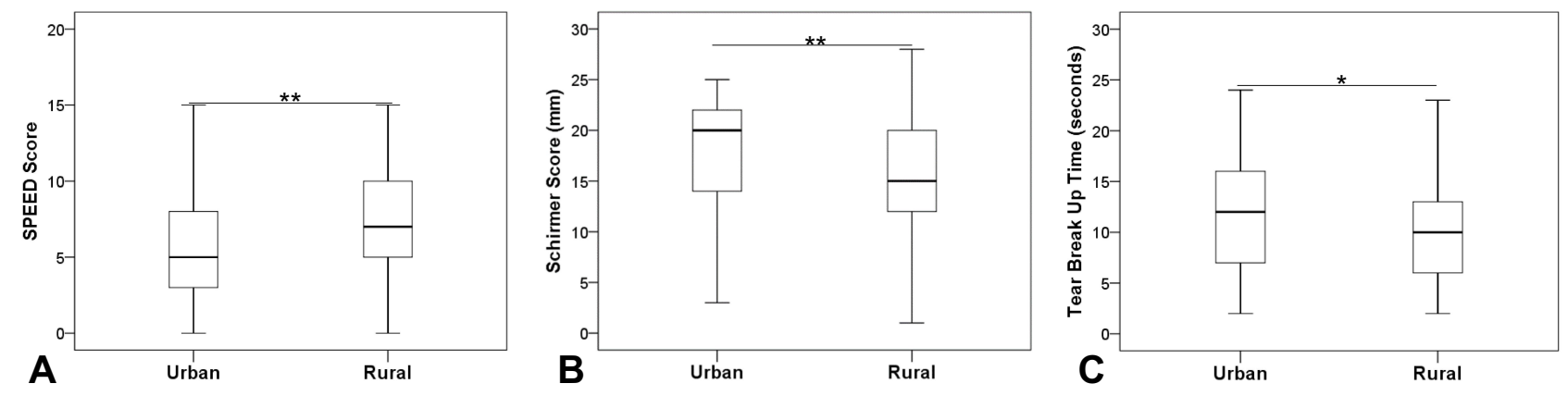

Figure 3 Differences in (A) SPEED scores, (B) Schirmer scores and (C) TBUT scores $\left({ }^{*} p<0.05\right.$, $\left.{ }^{* *} \mathrm{p}<0.0 \mathrm{I}\right)$.
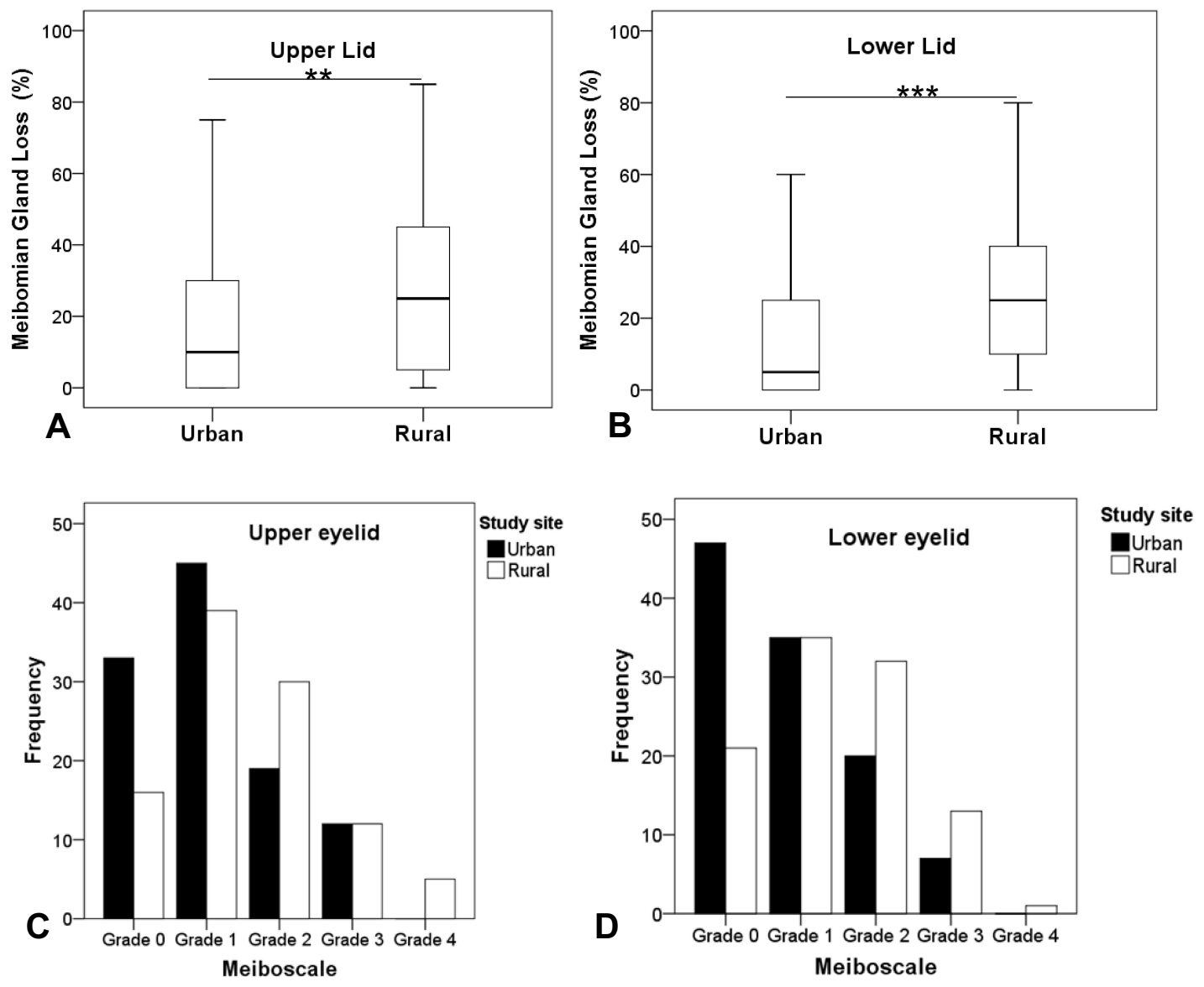

Figure 4 Differences in meibomian gland loss. (A) Upper lids and (B) lower lids (**p<0.0I, ${ }^{* * *} p<0.00$ I); differences in meiboscale distribution. (C) Upper lids and (D) lower lids.

MGL, $p<0.05$. Similarly, the odds of having MGL and a history of stye was higher for rural participants than urban participants. Rural participants with a history of stye had $12 \%$ higher odds ( $\mathrm{OR}=1.12$ [95\% CI, 0.38-3.27]) of having MGL, $p>0.05$ whereas urban participants with a history of stye had $18 \%$ lower odds (OR=0.82 [95\% CI, 0.33-2.10]) of having MGL, $\mathrm{p}>0.05$.

Rural participants scored higher on Pult's meiboscale (grades 2-4) than urban participants (grades 0 and
1). Chi-square test of association revealed statistically significant differences in meiboscale distributions between rural and urban participants. For the upper eyelids; $\chi^{2}(4, \quad \mathrm{~N}=211)=13.58, \mathrm{p}<0.01$ and for the lower eyelids; $\chi^{2}(4, \mathrm{~N}=211)=15.29, \mathrm{p}<0.01 \quad$ (Figure $4 \mathrm{C}$ and $\mathrm{D})$. The associations were statistically significant, positive and moderately strong for upper eyelid: $\varphi_{\mathrm{c}}=0.254, \mathrm{p}<0.01$ and lower $\operatorname{lid}_{\varphi_{\mathrm{c}}}=0.270, \mathrm{p}<0.01$, respectively. 


\section{Relationship Between Meibomian Gland Loss and Clinical Dry Eye Parameters}

Overall, we observed statistically significant correlation between age and MGL $\left[\mathrm{r}_{\mathrm{s}}=0.61, \mathrm{p}<0.01\right]$ and between MGL and OSS $\left[\mathrm{r}_{\mathrm{s}}=0.35, \mathrm{p}<0.01\right]$ (Figure $5 \mathrm{~A}$ and B). MGL was also significantly correlated with TBUT $\left[\mathrm{r}_{\mathrm{s}}=-0.52\right.$, $\mathrm{p}<0.01]$, and Schirmer scores $\left[\mathrm{r}_{\mathrm{s}}=-0.40, \mathrm{p}<0.01\right]$. These significant relationships did not change when age was controlled for. On the other hand, MGL and SPEED scores were not significantly correlated (Figure 5C-E).

\section{Discussion}

The results suggest there are significant differences in the clinical dry eye profile and meibomian gland health status between rural and urban study participants. Specifically, rural participants showed greater DED symptoms and signs (i.e., decreased tear film production and stability) than urban participants. Additionally, compared to urban participants, rural participants showed a greater proportion and increased severity (grades) of MGL. MGL is a major feature of MGD, which is a well-known cause of evaporative DED. MGD can significantly affect the severity of DED. ${ }^{1,3}$ Thus, it suffices to say that degree of MGL among all the participants, with more among the rural participants may be a major contributor to differences in DED profiles observed between the rural and urban participants.

Interestingly, we also found for the second time that the proportion of MGD patients in the Ghanaian population is higher than that reported in other major studies conducted outside of the Africa. ${ }^{11}$ Overall, we report $72.5 \% \%$ (rural $=82.3 \%$ and urban $=63.3 \%$ ) of the study participants had MGD, which is higher than reported in the Japanese Studies (61.8\%), Beijing Eye Study (69.3\%), the Salisbury Eye Evaluation Study (3.5\%) and Melbourne Visual Impairment Study (19.9\%). ${ }^{17-21}$ However, these participants were all symptomatic for DED - necessitating a larger epidemiological study which will include asymptomatic group of subjects. This will provide the closest insight into the prevalence of MGD in the Ghanaian population. It is also important to mention that the variations in reported prevalence values across these studies may partly be due to differences in diagnostic criteria for MGD and/or study designs. ${ }^{21}$ Since our study participants were all symptomatic for DED; presenting with one or more DED symptoms, and MGD was present among the majority it suggests MGD could be a primary contributor to their DED status. ${ }^{1,3,11}$

The link between MGD and DED has been well described. MGD involves chronic diffuse abnormality of
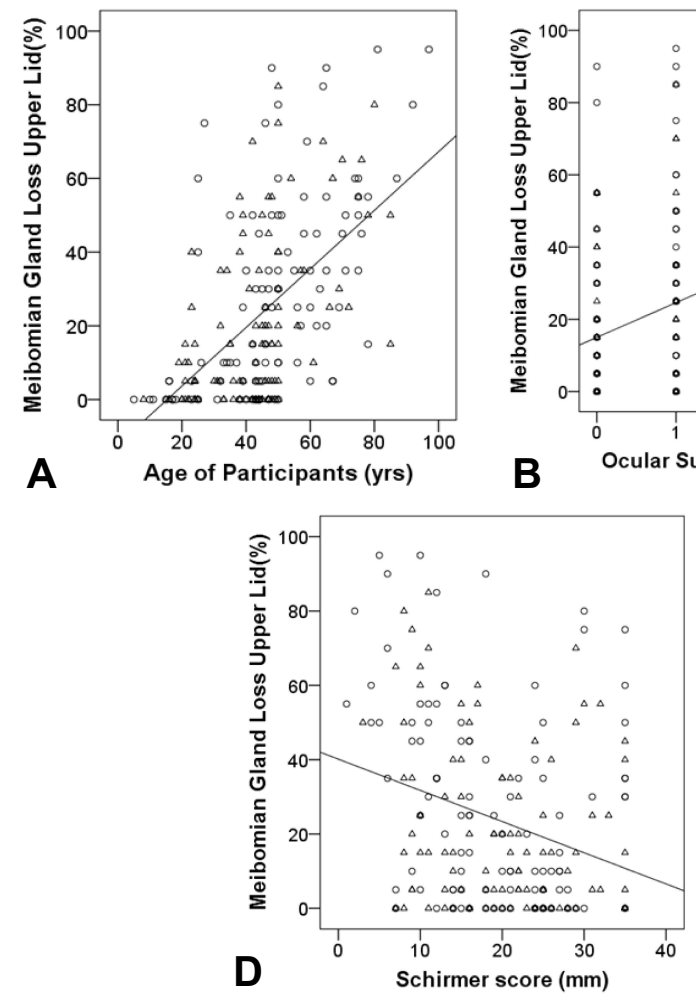
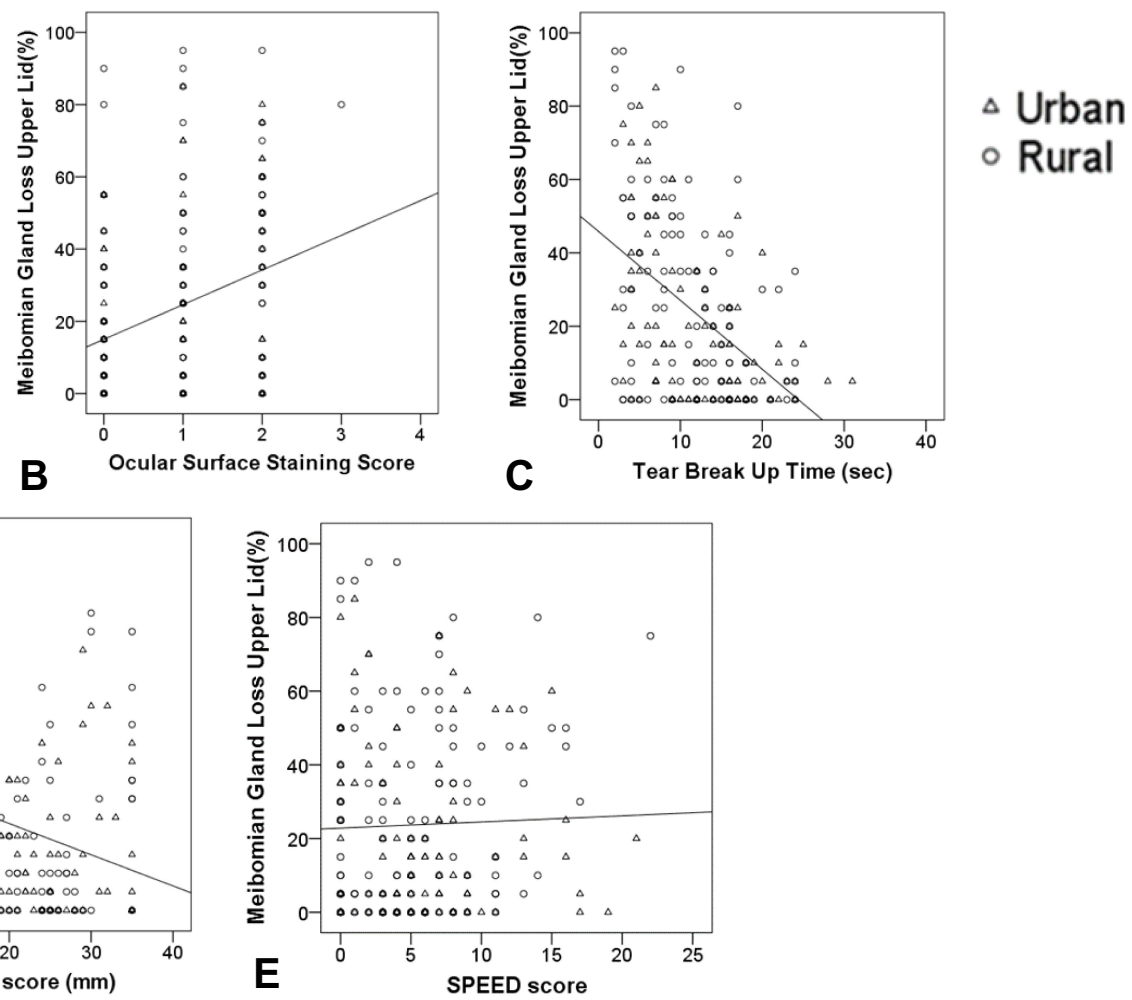

Figure 5 Overall correlation between meibomian gland loss and (A) age, (B) ocular surface staining, (C) tear break-up time, (D) Schirmer scores and (E) SPEED scores. 
the meibomian gland. ${ }^{1,3}$ It often includes qualitative and quantitative alterations in meibomian gland secretion (meibum) and structural changes (atrophy or loss) of the glands. ${ }^{1,3}$ It is also commonly characterised by terminal duct obstruction with resultant alteration of the tear film, symptoms of eye irritation, clinically apparent inflammation, and ocular surface disease. ${ }^{1,3}$ Meibum is critical for tear film stability, lubrication and thought to prevent infection at the ocular thereby preventing DED and ocular surface damage. ${ }^{3,22}$

Consequently, changes in the meibomian gland thus meibum can result in symptoms of dry eye as reported by our study participants. ${ }^{3,22}$ In fact, we recorded overall significant correlations between MGL and various indicators of DED such as decreased tear break-up time and tear production, and increased symptom (SPEED) scores. Similar observations have been reported in other studies. ${ }^{3,11,23}$ We also observed a significant correlation between MGL and age. Age is a risk factor for both MGD and DED, but it is important to mention that even after controlling for the effect of age, the significant relationships between MGL and the aforementioned DED indicators remained the same. ${ }^{3,21}$ This suggests that for this group of participants, the contribution of MGD to and its relationship with the observed DED states were independent of age. MGL however did not correlate with SPEED scores and it has been reported by other studies that DED signs and symptoms do always correlate with each other. ${ }^{24}$

The pathomechanism of MGD is complex and not fully understood. Different studies have mentioned the role of prolonged contact lenses, ageing, autoimmune disorders and the influence of environment. ${ }^{3,21,25}$ In particular, low humidity, high temperatures and desiccating conditions are believed to influence MGD and DED. Ghana being semiarid and in the tropical-savannah zone of Africa has these typical climatic conditions prevailing over a greater part of the year (Figure 1). ${ }^{26}$ However, it is important to mention that while all participants (rural and urban) come from the same region and experience the same climatic conditions rural participants did show an increased presence and severity of MGD and DED.

We believe the subtle differences in their everyday environment could explain the differences in the distribution of MGD and the severity of DED between the groups. For example, we observed a difference in occupational profiles of these participants with majority of the urban participants working in the formal sector, whereas majority of the rural subjects worked as farmers. Thus, the two groups of study participants are exposed to relatively different daily working and living environmental conditions which can impact their ocular and general health differently.

There also exists a complex relationship between health status and socioeconomic status. ${ }^{27,28}$ Most rural communities in Ghana lack access to basic amenities including clean water for drinking or washing, making it challenging for them to observe stricter patterns of personal hygiene. ${ }^{27-29}$ This can make rural participants more susceptible to microbial infections of the eyelids. And given that there is a crosstalk between eyelid dysbiosis, MGD, and DED this may explain why the rural participants showed severe MGL and DED symptoms. ${ }^{3,21,28,30}$

Additionally, there is a huge disparity in health/eye care services provision and utilization patterns between rural and urban areas in Ghana. ${ }^{29,31}$ Even where available, most rural inhabitants often delay in seeking treatment for their conditions due to financial constraints and in a few cases, hesitation to accept to orthodox medicine. Generally, most inhabitants of rural areas may seek hospital treatment only when their conditions have reached advanced stages. ${ }^{31,32}$ Similarly in this study, the rural participants were more symptomatic for DED and showed severe MGD than the urban participants because they perhaps presented for clinical evaluation and treatment only when their condition had advanced with intolerable symptoms.

In contrast to rural areas, industrialized and urban cities like Kumasi have increased problems with pollutants such as high environmental levels of nitrogen dioxide $\left(\mathrm{NO}_{2}\right)$, sulphur dioxide $\left(\mathrm{SO}_{2}\right)$, carbon monoxide $(\mathrm{CO})$ and ozone $\left(\mathrm{O}_{3}\right)$, and particulate matter (PMs) which can affect ocular surface health. Studies conducted in China and Korea implicate these environmental pollutants in the development of ocular surface conditions such as pterygium and DED. $\mathrm{SO}_{2}, \mathrm{O}_{3}$ and $\mathrm{PM}_{2.5}$ in particular have been named as important risk factors for DED. ${ }^{33-35}$

Furthermore, as it is very typical of most developed and emerging economies, urban living in Ghana is also characterized by longer "screen times" - with prolonged use of smartphones and computers at home and work. Most people in urban Ghana may also spend longer hours working or living in air-conditioned environments. ${ }^{1,4,6,12}$ While we did not characterize these factors in this study, the results show that occupation differed between both groups with majority of urban participants working in the 
civil service where computer and air-conditioner use is common. It is therefore possible that for urban participants, the frequent exposure of the ocular surface to these adverse environmental conditions could have contributed to their DED. ${ }^{1,6,11-13}$

Moreover, with regards to hazardous working/environmental conditions, farmers in rural Ghana may come in contact with vegetative or soil materials that could impact the ocular surface and adnexa by way of injury or infection. Majority of the rural participants were farmers and our results show the cases of stye and blepharitis, which are two common eyelid conditions linked to MGD were two and six times higher, respectively, among rural participants. As observed, the rural participants showed increased odds of the having a history of stye or blepharitis and having MGL than urban participants.

It is important to mention that although the differences in between the exposure arms (blepharitis vs nonblepharitis, and stye vs non-stye) were insignificant for rural participants, the very high percentage of MGL among rural subjects cannot be refuted as clinically unmeaningful. This is because, recurrent blepharitis and stye have been reported to affect the meibomian glands, leading to MGD hence evaporative DED. Several reports also mention a close association between these eyelid conditions and DED. ${ }^{3,21,29}$ Thus, the observation of a statistically insignificant difference between the exposure arms for the rural participants may be due to inherent sample characteristics; for example, our sample was not normally distributed.

\section{Conclusions}

In summary, the study presents relevant information about DED and Meibomian gland health status in Ghana by comparing and highlighting significant differences in the DED and MGD profiles between participants from rural and urban Ghanaian populations. The significant relationships between the various clinical variables suggest important links between MGD and DED with likely contributions from the participants' daily and working environments. Our findings thus demonstrate that even in a region where climatic conditions are the same, subtle differences in the daily living and working environments including the potential role of environmental pollutants in urban areas or hygiene conditions in rural areas, and a history of eyelid disease can influence the occurrence and severity of MGD and DED.

\section{Acknowledgments}

The authors are grateful to Benjamin Ghansah at the International Water Management Institute - Ghana for helping generate the maps in this paper. Drs Kwame Amankwah Kwarteng and Ernest Kyei Nkansah also supported data collection at the Westphalian Eye Hospital.

\section{Funding}

During the time of the study, Philipp Steven had a Deutsche Forschungsgemeinschaft Forschergruppe FOR 2240, DFG STE 1928/4-1 grant.

\section{Disclosure}

The authors report no conflicts of interest in this work.

\section{References}

1. Craig JP, Nichols KK, Akpek EK, et al. TFOS DEWS II definition and classification report. Ocul Surf. 2017;15(3):276-283. doi:10.10 16/j.jtos.2017.05.008

2. Lam H, Bleiden L, De Paiva CS, Farley W, Stern ME, Pflugfelder SC. Tear cytokine profiles in dysfunctional tear syndrome. Am J Ophthalmol. 2009;147(2):198-205. e1. doi:10.1016/j.ajo.2008.08. 032

3. Knop E, Knop N, Millar T, Obata H, Sullivan DA. The international workshop on meibomian gland dysfunction: report of the subcommittee on anatomy, physiology, and pathophysiology of the meibomian gland. Invest Ophthalmol Vis Sci. 2011;52(4):1938-1978. doi:10.1167/iovs.10-6997c

4. Stapleton F, Alves M, Bunya VY, et al. TFOS DEWS II epidemiology report. Ocul Surf. 2017;15(3):334-365. doi:10.1016/j.jtos.2017. 05.003

5. Tahmaz V, Gehlsen U, Sauerbier L, et al. Treatment of severe chronic ocular graft-versus-host disease using $100 \%$ autologous serum eye drops from a sealed manufacturing system: a retrospective cohort study. Br J Ophthalmol. 2017;101(3):322-326.

6. Osae A, Gehlsen U, Horstmann J, et al. Epidemiology of dry eye disease in Africa: the sparse information, gaps and opportunities. Ocul Surf. 2017;15(2):159-168. doi:10.1016/j.jtos.2017.01.001

7. Moore DB, Shirefaw W, Tomkins-Netzer O, Eshete Z, NetzerTomkins H, Ben-Zion I. Prevalence of xerophthalmia among malnourished children in rural Ethiopia. Int Ophthalmol. 2013;33 (5):455-459. doi:10.1007/s10792-013-9715-9

8. Schémann J-F, Malvy D, Sacko D, Traore L. Trachoma and vitamin A deficiency. Lancet. 2001;357(9269):1676. doi:10.1016/S0140-6736 (00)04833-9

9. Burki T. The broad benefits of trachoma elimination. Lancet Infect Dis. 2016;16(5):530-531. doi:10.1016/S1473-3099(16)30017-2

10. Mayo-Wilson E, Imdad A, Herzer K, Yakoob MY, Bhutta ZA. Vitamin A supplements for preventing mortality, illness, and blindness in children aged under 5: systematic review and meta-analysis. BMJ. 2011;343(aug25 1):d5094. doi:10.1136/bmj.d5094

11. Osae EA, Ablorddepey RK, Horstmann J, Kumah DB, Steven P. Assessment of meibomian glands using a custom-made meibographer in dry eye patients in Ghana. BMC Ophthalmol. 2018;18(1):201. doi:10.1186/s12886-018-0869-0

12. Cobbinah PB, Amoako C. Urban sprawl and the loss of peri-urban land in Kumasi, Ghana. Int J Soc Human Sci. 2012;6(388):e397. 
13. Oduro CY, Ocloo K, Peprah C. Analyzing growth patterns of Greater Kumasi metropolitan area using GIS and multiple regression techniques. J Sustain Dev. 2014;7(5):13. doi:10.5539/jsd.v7n5p13

14. Ngo W, Situ P, Keir N, Korb D, Blackie C, Simpson T. Psychometric properties and validation of the standard patient evaluation of eye dryness questionnaire. Cornea. 2013;32(9):1204-1210. doi:10.1097/ ICO.0b013e318294b0c0

15. Bron AJ, Evans VE, Smith JA. Grading of corneal and conjunctival staining in the context of other dry eye tests. Cornea. 2003;22 (7):640-650. doi:10.1097/00003226-200310000-00008

16. Pult H, Riede-Pult B. Non-contact meibography: keep it simple but effective. Cont Lens Anterior Eye. 2012;35(2):77-80. doi:10.1016/j. clae.2011.08.003

17. Jie Y, Xu L, Wu Y, Jonas J. Prevalence of dry eye among adult Chinese in the Beijing Eye Study. Eye. 2009;23(3):688-693. doi:10.1038/sj.eye.6703101

18. Uchino M, Dogru M, Yagi Y, et al. The features of dry eye disease in a Japanese elderly population. Optom Vis Sci. 2006;83(11):797-802. doi:10.1097/01.opx.0000232814.39651.fa

19. Schein OD, MUÑO B, Tielsch JM, Bandeen-Roche K, West S. Prevalence of dry eye among the elderly. Am J Ophthalmol. 1997;124(6):723-728. doi:10.1016/S0002-9394(14)71688-5

20. McCarty CA, Bansal AK, Livingston PM, Stanislavsky YL, Taylor HR. The epidemiology of dry eye in Melbourne, Australia. Ophthalmology. 1998;105(6):1114-1119. doi:10.1016/S0161-6420 (98)96016-X

21. Schaumberg DA, Nichols JJ, Papas EB, Tong L, Uchino M, Nichols KK. The international workshop on meibomian gland dysfunction: report of the subcommittee on the epidemiology of, and associated risk factors for, MGD. Invest Ophthalmol Vis Sci. 2011;52(4):1994 2005. doi:10.1167/iovs.10-6997e

22. Millar TJ, Schuett BS. The real reason for having a meibomian lipid layer covering the outer surface of the tear film-a review. Exp Eye Res. 2015;137:125-138. doi:10.1016/j.exer.2015.05.002

23. Mathers WD. Ocular evaporation in meibomian gland dysfunction and dry eye. Ophthalmology. 1993;100(3):347-351. doi:10.1016/ S0161-6420(93)31643-X

24. Nichols KK, Nichols JJ, Mitchell GL. The lack of association between signs and symptoms in patients with dry eye disease. Cornea. 2004;23(8):762-770. doi:10.1097/01.ico.0000133997.0714 $4.9 \mathrm{e}$

Clinical Ophthalmology

\section{Publish your work in this journal}

Clinical Ophthalmology is an international, peer-reviewed journal covering all subspecialties within ophthalmology. Key topics include: Optometry; Visual science; Pharmacology and drug therapy in eye diseases; Basic Sciences; Primary and Secondary eye care; Patient Safety and Quality of Care Improvements. This journal is indexed on PubMed

Submit your manuscript here: https://www.dovepress.com/clinical-ophthalmology-journal
25. Engel L, Wittig S, Bock F, et al. Meibography and meibomian gland measurements in ocular graft-versus-host disease. Bone Marrow Transplant. 2015;50(7):961-967. doi:10.1038/bmt.2015.72

26. Stanturf J, Warren M, Charnley S, et al. Ghana Climate Change Vulnerability and Adaptation Assessment. Washington: United States Agency for International Development; 2011.

27. Novignon J, Nonvignon J, Mussa R, Chiwaula LS. Health and vulnerability to poverty in Ghana: evidence from the Ghana living standards survey round 5. Health Econ Rev. 2012;2(1):11. doi:10.1186/2191-1991-2-11

28. Rotondo LA, Ngondi J, Rodgers AF, et al. Evaluation of community intervention with pit latrines for trachoma control in Ghana, Mali, Niger and Nigeria. Int Health. 2009;1(2):154-162. doi:10.1016/j. inhe.2009.08.001

29. Pengpid S, Peltzer K. Hygiene behaviour and health attitudes in African countries. Curr Opin Psychiatry. 2012;25(2):149-154. doi:10.1097/YCO.0b013e32834fda33

30. Zhang SD, He JN, Niu TT, et al. Bacteriological profile of ocular surface flora in meibomian gland dysfunction. Ocul Surf. 2017;15 (2):242-247. doi:10.1016/j.jtos.2016.12.003

31. Ocansey S, Kumi-Kyereme A, Awusabo-Asare K, Ilechie AA, BoadiKusi SB, Abraham CH. Utilization of eye care services among Ghanaian elderly population: evidence from a peri-urban community. Ophthalmol Res. 2013;89-101.

32. Tipping G, Segall M. Health care seeking behaviour in developing countries. An annotated bibliography and literature review. 1995.

33. Bortey-Sam N, Ikenaka Y, Akoto O, et al. Levels, potential sources and human health risk of polycyclic aromatic hydrocarbons (PAHs) in particulate matter (PM 10) in Kumasi, Ghana. Environ Sci Pollut Res. 2015;22(13):9658-9667. doi:10.1007/s11356-014-4022-1

34. Obeng G, Mensah E, Ashiagbor G, Boahen O, Sweeney D. Watching the smoke rise up: thermal efficiency, pollutant emissions and global warming impact of three biomass cookstoves in Ghana. Energies. 2017;10(5):641. doi:10.3390/en10050641

35. Lee KW, Choi Y-H, Hwang SH, et al. Outdoor air pollution and pterygium in Korea. $J$ Korean Med Sci. 2017;32(1):143-150. doi:10.3346/jkms.2017.32.1.143
Central and CAS, and is the official journal of The Society of Clinical Ophthalmology (SCO). The manuscript management system is completely online and includes a very quick and fair peer-review system, which is all easy to use. Visit http://www.dovepress.com/ testimonials.php to read real quotes from published authors. 